\title{
Adaptive Classification of Dirt Particles in Papermaking Process
}

\author{
Nataliya Strokina, Tuomas Eerola, Lasse Lensu, and Heikki Kälviäinen \\ Machine Vision and Pattern Recognition Laboratory (MVPR) \\ Department of Information Technology \\ Lappeenranta University of Technology (LUT) \\ P.O. Box 20, FI-53851 Lappeenranta, Finland \\ firstname.surname@lut.fi \\ http://www2.it.lut.fi/mvpr
}

\begin{abstract}
In pulping and papermaking, dirt particles significantly affect the quality of paper. Knowledge of the dirt type helps to track the sources of the impurities which would considerably improve the paper making process. Dirt particle classification designed for this purpose should be adaptable because the dirt types are specific to the different processes of paper mills. This paper introduces a general approach for the adaptable classification system. The attention is paid to feature extraction and evaluation, in order to determine a suboptimal set of features for a certain data. The performance of standard classifiers on the provided data is presented, considering how the dirt particles or different types are classified. The effect of dirt particle grouping according to the particle size on the results of classification and feature evaluation is discussed. It is shown that the representative features of dirt particles from different size groups are different, which has an effect on the classification.
\end{abstract}

Keywords: machine vision, particle segmentation, dirt particle classification, feature extraction, pulping, papermaking, image processing and analysis.

\section{Introduction}

Recently the papermaking industry has been focusing on process optimization and has become more interested in machine vision methods. Dirt particles affect considerably the formation of paper, impairing the printing properties. Tracking the amount and type of dirt in pulp enables to use the materials and energy more efficiently. Additionally, knowing the type of the particles it is easier to determine the source of dirt and to eliminate the problem of dirt particles. Manual dirt counting is a time-consuming process, involving human for analysis and evaluation. In this case the human factor affects significantly the results of evaluation. A human is not able to keep the same level of concentration and attention during the whole period of work, and the decisions might be subjective depending on a researcher. The process of dirt counting might be automated so that

A. Heyden and F. Kahl (Eds.): SCIA 2011, LNCS 6688, pp. 731-741, 2011.

(C) Springer-Verlag Berlin Heidelberg 2011 
the tedious work is performed by a machine and a human has only to analyze the results.

The machine vision approach in the paper quality control consists of acquisition of digital images of the paper samples and the analysis of the digitized samples using computational methods. The idea of such systems is described in $\begin{array}{lll}1 & 2 & 3\end{array}$ where not only off-line but also on-line methods for paper evaluation are presented. The off-line methods for dirt detection consider scanned images of the paper sheets. The system evaluates the mean intensity of the pixels and according to the local threshold segments the impurities. In [2] a threshold is determined manually, in the other cases there is an opportunity of automatic calculation of the threshold. On-line systems usually adapt to the intensity automatically and determine the threshold. The classification of particles is not done or only dirt clustering according to the shape is performed, e.g., in [6].

This study focuses on the development of the approach for adaptive classification of the dirt particles. The approach should use a standard classification method to be capable of adapting for the specific data. Types of dirt features and their evaluation are paid a special attention. The particles are divided into subgroups according to their size and the results of classification for the subgroups are compared to the results without the division. Section 2 provides the description of the approach and the method proposed. In Section 3 the results of feature extraction and classification are discussed. The conclusions are drawn in Section 4 .

\section{Classification of Dirt Particles}

\subsection{Problem Statement}

Objectives. The aim of the presented research is to develop a method for adaptive classification of dirt particles in dry pulp sheets. Feature selection should be automated. State-of-art generic classification methods are used so that the classification was not related to any data. It is important since the system should handle adding new dirt types. Additionally, it is needed to study how the division of dirt particles according to their size can affect the classification results.

Restrictions. The research is based only on the provided data, which includes dry pulp sheets with dirt particles of four dirt types: bark, sand, plastic, and shives. The provided data does not introduce all the possible variations of pulp and dirt.

Test samples. Each test sample contains dirt of a single type. The samples were scanned, and the examples of test samples are presented in Figure 1.

\subsection{General Workflow}

Algorithm 1 introduces the main steps of the adaptive classification. After dirt particle segmentation the grouping of the whole set based on the area is performed. 


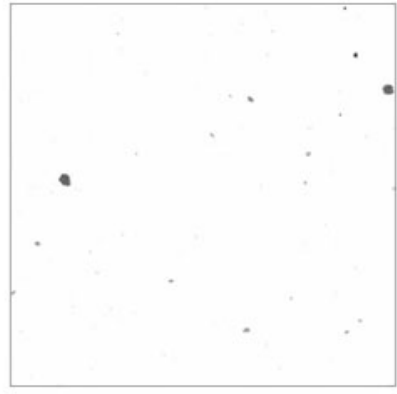

(a)

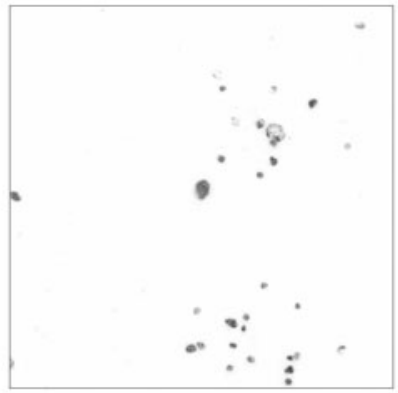

(c)

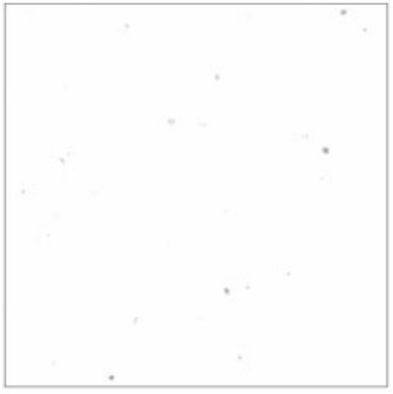

(b)

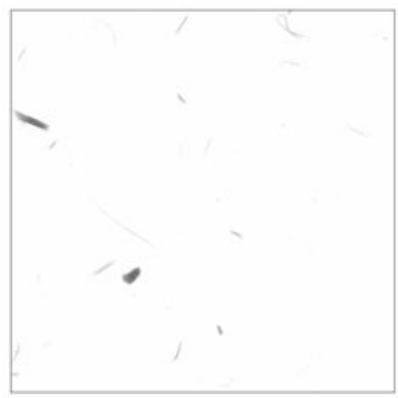

(d)

Fig. 1. Pulp sheets: (a) Bark; (b) Plastic; (c) Sand; (d) Shive

In the case of multiple subsets the features should be evaluated separately for each subset since for dirt particles of different size different characteristics can be significant. It is worth mentioning that the classification method should not be related to the specific data set since the available data does not represent all the possible dirt particles and pulp types. For these reasons the performance of several standard classifier is evaluated.

\subsection{Grouping of Dirt Particles Based on a Size}

The experiments have revealed that dirt particles of different size might have different features that count. Varying significantly within one class some features cause problems in classification because of considerable overlapping of the classes in feature space. A number of grounds might be found to figure out groups of particles within one class. For example, based on the area histogram one could divide one class into several depending on histogram bins. In the this work ISO 5350-1:2006 [13] is employed for group categorization. 


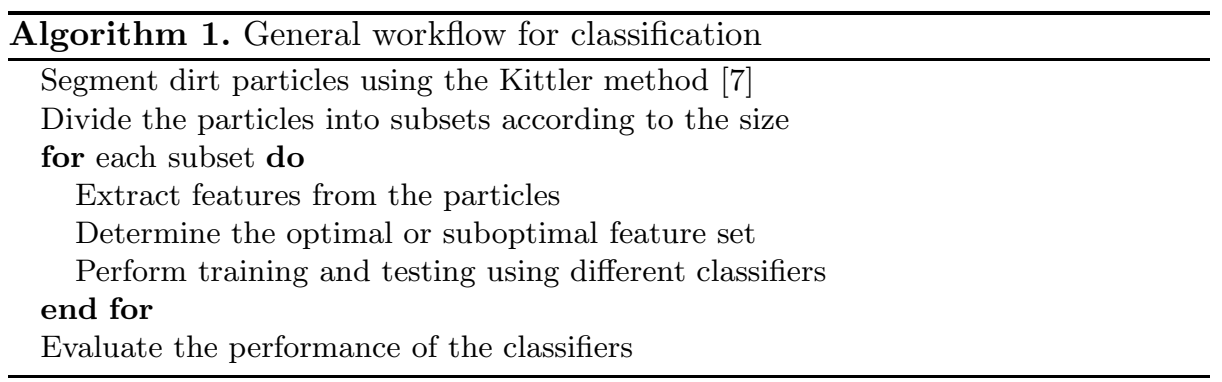

\subsection{Segmentation}

Segmentation of dirt particles is an important stage at which small low contrast areas should be detected in an image. According to the survey [10, there is a number of methods to use but none of them can be universally used for any segmentation problem. In this study the Kittler thresholding method [7] is used. The choice of the method is based on the previous study on automated counting and characterization of dirt particles in pulp 4 .

Grayscale images are considered for segmentation. The images are divided into foreground which consists of the dirt particles and background. The foreground and background are modeled as a mixture of two Gaussians [7. The threshold can be calculated by optimizing the cost function based on the Bayesian classification rule.

\subsection{Feature Extraction}

The features can be divided into two categories: geometric features and color features. Geometric features include characteristics of shape, form, and uniformity of a dirt particle. Color features include, for example, mean color, variation of color, and intensity. The calculated features are presented in Table 1

Table 1. Feature set

$\begin{array}{lllll}\text { Maximum diameter } & \text { Extent } & & \text { Elongation } & \text { Eq. (6) } \\ \text { Minimum diameter } & \text { Fiber length } & \text { Eq. (1) } & \text { Curl } & \text { Eq. (17) } \\ \text { Solidity } & \text { Fiber width } & \text { Eq. (2) } & \text { Mean intensity } & \\ \text { Eccentricity } & \text { Form factor } & \text { Eq. (3) } & \text { Mean color } & \\ \text { Convex area } & \text { Roundness } & \text { Eq. (44) } & \text { Area } & \\ \text { Perimeter } & \text { Aspect ratio } & \text { Eq. (5) } & \text { Coarseness } & \text { Eq. (8) }\end{array}$

Std of color

For each dirt particle a bounding box is determined which is the smallest rectangle enclosing the dirt particle. The Solidity specifies the proportion of the pixels in the convex hull that are also in the region. The Eccentricity specifies the eccentricity of the ellipse that contains the same second-moments as the region. Convex area is the number of pixels in the convex hull of a dirt particle. 
Extent specifies the ratio of pixels in the region to pixels in the total bounding box. Mean color and mean intensity are calculated as the mean hue value and the mean intensity over a dirt particle area. Std of color describes the standard deviation of color within the area of a dirt particle.

Other geometric features are calculated according to the following formulas:

$$
\begin{gathered}
\text { Fiberlength }=0.25 \cdot\left(\text { Perimeter }-\left(\sqrt{\mid \text { Perimeter }^{2}-16 \cdot \text { Area } \mid}\right)\right) \\
\text { FiberWidth }=\frac{\text { Area }}{\text { FiberLength }} \\
\text { FormFactor }=\frac{4 \cdot \pi \cdot \text { Area }^{\text {Perimeter }}}{\text { Roundness }=\frac{4 \cdot \text { Area }^{2}}{\pi \cdot \text { MaxDiameter }}{ }^{2}} \\
\text { AspectRatio }=\frac{\text { MaxDiameter }}{\text { MinDiameter }} \\
\text { Elongation }=\left|\frac{\text { FiberLength }}{\text { FiberWidth }}\right| \\
\text { Curl }=\left|\frac{\text { MaxDiameter }}{\text { FiberLength }}\right| \\
\text { Coarseness }=\frac{\text { Perimeter }}{4 \cdot \pi \cdot \text { Area }^{2}}
\end{gathered}
$$

\subsection{Feature Evaluation}

Feature evaluation is performed to determine the set of features that should be used to classify the dirt particles. The evaluation function is determined by the Linear Discriminant Analysis (LDA) 5] that is used for data classification and the reduction of feature space dimensions.

The goal of the LDA method is to maximize the separability of data classes, which implies the maximization of the ratio

$$
r=\operatorname{tr}\left(\frac{\left|S_{b}\right|}{\left|S_{w}\right|}\right) .
$$

Within the class scatter matrix

$$
S_{w}=\sum_{i=1}^{C} \sum_{j=1}^{N_{i}}\left(x_{j}-\text { mean }_{i}\right)
$$

where $C$ is the number of classes, $N_{i}$ is the number of samples in class $i, x_{j}$ is the $\mathrm{j}$-th element of class $i$, and mean $_{i}$ is the mean value of class $i$, determines the variance of values within one class. 
Between the class scatter matrix

$$
S_{b}=\sum_{i=1}^{C}\left(\text { mean }_{i}-\text { mean }\right)
$$

where mean is the mean of the means of all the classes, evaluates the variance of feature values within one class.

To find the space where the current features might be distinguishable in the most efficient way one should maximize the criterion

$$
c r=\operatorname{tr}\left(S_{w}^{-1} \cdot S_{b}\right)
$$

which means that the eigenvectors of the criterion matrix should be calculated. The matrix of eigenvectors describes the transformation to the new feature space. The sample vectors from all the classes should be transferred to that space and using one or several thresholds might be distinguishable.

In order to decrease the computational time suboptimal algorithms are usually employed. An algorithm should be developed specifically for a certain task. A sequential approach for feature selection is presented in Algorithm 2. In this case the ratio is calculated for different combinations of features, consisting of one, two, and three items. If the optimal combination consists of three features, the values of ratio for the combinations consisting of the optimal set and one new feature is calculated. If the current ratio is more than the maximum, one should continue adding features until the calculated ratio is less than the maximum. As an output the algorithm provides the suboptimal feature set and the ratio value for it.

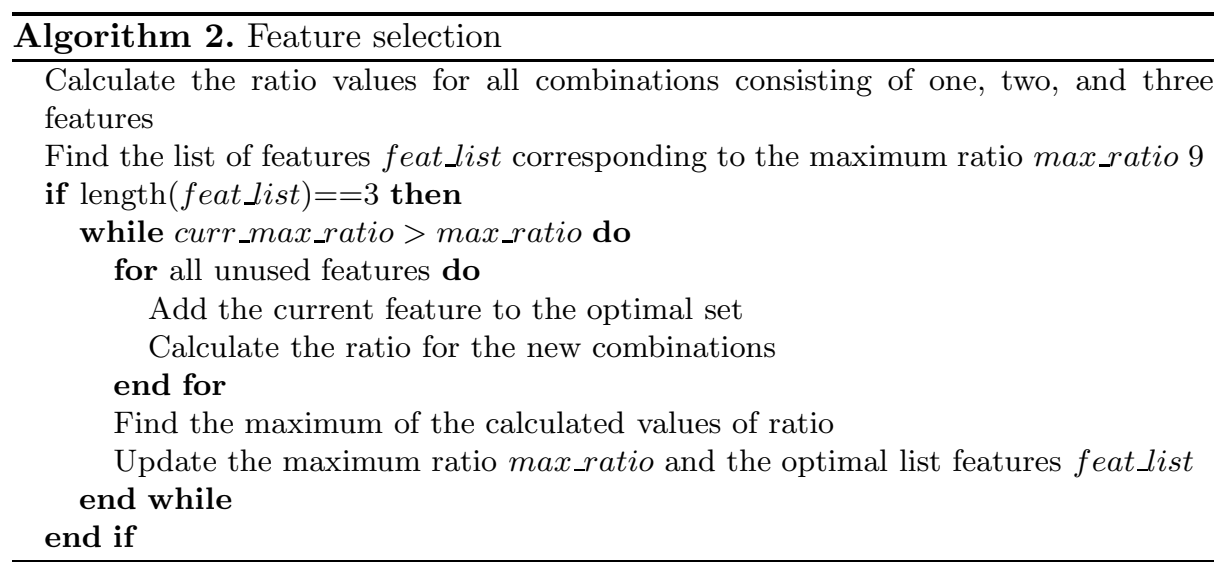

\subsection{Classification}

The classifiers used in the study are listed in Table 2. State-of-the-art generic classification methods as well as the well-known structural approaches are used in order not to be related to the specific data. K-NN is used with a neighborhood of 1,3 , and 5 samples. LDA is based on the transformation described in 
Section 2, where the linearizing transformation is described. GMM classifier is used with expectation maximization (GMMem) and Figueiredo-Jain (GMMfj) criteria [8. SVM is used with a second order polynomial kernel. In the case of expectation maximization criterion 4 components were used to model the data. For Figueiredo-Jain criterion the maximum number of components was 10 . The Adaboost method introduces a set of weak classifiers, represented as a separate feature, to create a strong classifier. In the case of the Adaboost classification all the features are employed.

Table 2. List of classifiers

\begin{tabular}{|c|c|}
\hline K-Nearest Neighborhood (k-NN) 9 & Gaussian Mixture Model (GMM) \\
\hline Naive Bayesian Classifier (Bayes) 9 & Support Vector Machine (SVM) \\
\hline Discriminant & AdaBoost \\
\hline
\end{tabular}

\section{Experiments and Discussion}

\subsection{Test Samples and Experiments}

The samples for experiments are prepared by papermaking specialists of LUT FiberLaboratory. Based on the expert choice the selected types of particles are plastic, bark, sand, and shive. The samples have been scanned with reflective light. The examples of dirt particles used in the experiments are presented in Figure 1. The examples of the separate dirt particles are presented in Figure 2.

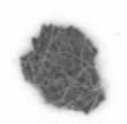

(a)

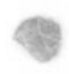

(b)

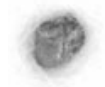

(c)

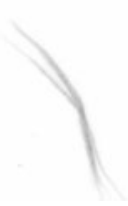

(d)

Fig. 2. Dirt particles: (a) Bark; (b) Plastic; (c) Sand; (d) Shive

The dirt particles were segmented and features were computed using Algorithm 2. Since the amount of dirt particles was restricted two middle size groups were combined into one. The suboptimal feature sets for each size group are described in Table 3 .

From the results it can be seen that the color is more important for bigger particles, since the color of smaller ones is not saturated. Standard deviation of color also becomes a more effective when the area of particles is large enough to estimate the feature. At the same time coarseness is considered to be a significant feature for the smaller groups. It is interesting to notice, that for the whole set of 
Table 3. Suboptimal feature sets for each size group

\begin{tabular}{|l|c|}
\hline Group & Feature set \\
\hline $0.04-0.15 \mathrm{~mm}^{2}$ & Form factor + Coarseness \\
$0.15-1.00 \mathrm{~mm}^{2}$ & StdColor + Coarseness \\
$1.00-5.00 \mathrm{~mm}^{2}$ & Mean color + Std of color \\
\hline The whole set & Mean color + Std of color \\
\hline
\end{tabular}

particles, without division into groups, the most effective features are the mean color and the standard deviation of color as for the biggest particles.

Two experiments were carried out. In the first experiment the whole data set was considered and the classification was performed on the training set, on the separate test set and using leave-one-out validation. In the second experiment the dirt particles were divided into the size groups. The classification was performed for all subsets separately. Table [5 shows the amount of particles in the train and the test sets for each dirt type for the whole set and for the size groups. The particles for training and testing were selected independently for the size groups and for the whole set. The amount of the dirt particles in the test set was restricted by the total amount of dirt.

\subsection{Classification on the Whole Set}

The classification results can be found in Table 4. The numbers represen the percentage of correctly classified dirt particles. For better representation the highest results are set in bold. One can notice that it is possible to divide the classifiers into two groups according to their performance. The first group with the best performance includes nearest neighborhood methods, SVM, and Gaussian mixture model classifiers producing approximately the same result. The poor performance of LDA and Adaboost can be explained by the fact that the classes are significantly overlapping in the feature space. From the results one can conclude that both the choice of an appropriate classifier and the selection of the optimal or suboptimal features lead to the sufficient classification results.

\subsection{Classification Using Subsets}

The results of classification have been averaged for the size groups and can be found in Table 6. The numbers represent the percentage of correctly classified dirt particles.

The result of classification for size groups are slightly worse than for the whole set which can be conditioned by the lack of samples in each of the groups. Here the same tendency among classification methods can be noticed as in the experiment with the whole set. The classifiers giving the best results are the Gaussian mixture model approach and the nearest neighborhood classifiers. However, the Adaboost, SVM and LDA classifiers have better performance than in the case of the whole set, which means that the size groups of particles are less overlapping in the feature space than the whole set. 
Table 4. Classification results for the whole set

\begin{tabular}{|l|c|c|c|c|c|c|c|}
\hline \multirow{2}{*}{ Classifier } & \multirow{2}{*}{ Train set } & \multirow{2}{*}{ Leave-one-out } & \multicolumn{5}{|c|}{ Test set } \\
\cline { 4 - 8 } & & & Bark & Plastic & Sand & Shive & Average \\
\hline 1-NN & - & $\mathbf{9 8 . 3 \%}$ & $87.6 \%$ & $97.5 \%$ & $88.7 \%$ & $64.4 \%$ & $\mathbf{8 3 . 3 \%}$ \\
\hline $3-\mathrm{NN}$ & $98.0 \%$ & $97.6 \%$ & $86.7 \%$ & $96.8 \%$ & $84.4 \%$ & $55.9 \%$ & $82.1 \%$ \\
\hline $5-\mathrm{NN}$ & $\mathbf{9 8 . 9 \%}$ & $96.6 \%$ & $\mathbf{9 5 . 2 \%}$ & $\mathbf{9 8 . 2 \%}$ & $54.6 \%$ & $51.7 \%$ & $82.4 \%$ \\
\hline Bayes & $87.0 \%$ & $85.3 \%$ & $74.5 \%$ & $81.2 \%$ & $29.7 \%$ & $65.2 \%$ & $68.8 \%$ \\
\hline LDA & $68.3 \%$ & $67.0 \%$ & $92.1 \%$ & $51.6 \%$ & $79.4 \%$ & $66.1 \%$ & $59.8 \%$ \\
\hline GMMfj & $97.3 \%$ & $93.7 \%$ & $84.5 \%$ & $96.0 \%$ & $85.1 \%$ & $45.5 \%$ & $78.8 \%$ \\
\hline GMMem & $98.5 \%$ & $96.0 \%$ & $83.8 \%$ & $95.5 \%$ & $51.1 \%$ & $45.0 \%$ & $77.3 \%$ \\
\hline SVM & $81.3 \%$ & $78.5 \%$ & $82.2 \%$ & $84.7 \%$ & $\mathbf{9 7 . 3 \%}$ & $40.0 \%$ & $76.1 \%$ \\
\hline AdaBoost & $73.3 \%$ & $69.5 \%$ & $54.6 \%$ & $82.6 \%$ & $57.6 \%$ & $\mathbf{7 9 . 4 \%}$ & $68.8 \%$ \\
\hline
\end{tabular}

Table 5. Amount of particles for each dirt type

\begin{tabular}{|c|c|c|c|c|c|c|c|c|}
\hline \multirow{2}{*}{ Size group } & \multicolumn{4}{|c|}{ Train set } & \multicolumn{4}{c|}{ Test set } \\
\cline { 2 - 9 } & Bark & Plastic & Sand & Shive & Bark & Plastic & Sand & Shive \\
\hline $0.04-0.15 \mathrm{~mm}^{2}$ & 50 & 50 & 50 & 50 & 609 & 89 & 29 & 22 \\
$0.15-1.00 \mathrm{~mm}^{2}$ & 20 & 20 & 20 & 20 & 342 & 184 & 9 & 55 \\
$1.00-5.00 \mathrm{~mm}^{2}$ & 40 & 40 & 40 & 40 & 20 & 24 & 24 & 101 \\
\hline The whole set & 100 & 100 & 100 & 100 & 981 & 307 & 72 & 188 \\
\hline
\end{tabular}

Table 6. Classification results for the size groups

\begin{tabular}{|l|c|c|c|c|c|c|c|}
\hline \multirow{2}{*}{ Classifier } & \multirow{2}{*}{ Train set } & \multirow{2}{*}{ Leave-one-out } & \multicolumn{5}{|c|}{ Test set } \\
\cline { 4 - 8 } & & & Bark & Plastic & Sand & Shive & Average \\
\hline 1-NN & - & $75.5 \%$ & $68.8 \%$ & $59.3 \%$ & $61.5 \%$ & $59.2 \%$ & $62.2 \%$ \\
\hline $3-\mathrm{NN}$ & $88.4 \%$ & $80.3 \%$ & $76.6 \%$ & $55.2 \%$ & $60.7 \%$ & $63.5 \%$ & $64.0 \%$ \\
\hline $5-\mathrm{NN}$ & $85.5 \%$ & $77.8 \%$ & $76.3 \%$ & $52.9 \%$ & $57.5 \%$ & $67.8 \%$ & $63.6 \%$ \\
\hline Bayes & $83.9 \%$ & $79.2 \%$ & $90.9 \%$ & $50.0 \%$ & $58.1 \%$ & $62.2 \%$ & $65.3 \%$ \\
\hline LDA & $65.6 \%$ & $64.8 \%$ & $84.2 \%$ & $42.3 \%$ & $70.9 \%$ & $61.6 \%$ & $64.8 \%$ \\
\hline GMMfj & $\mathbf{9 5 . 1 \%}$ & $83.0 \%$ & $77.5 \%$ & $68.8 \%$ & $64.3 \%$ & $64.0 \%$ & $68.7 \%$ \\
\hline GMMem & $89.5 \%$ & $\mathbf{8 6 . 3 \%}$ & $\mathbf{9 4 . 5 \%}$ & $70.1 \%$ & $49.1 \%$ & $64.5 \%$ & $\mathbf{6 9 . 5 \%}$ \\
\hline SVM & $67.8 \%$ & $64.3 \%$ & $75.6 \%$ & $\mathbf{7 3 . 3 \%}$ & $52.1 \%$ & $41.1 \%$ & $60.5 \%$ \\
\hline AdaBoost & $71.2 \%$ & $69.8 \%$ & $58.7 \%$ & $56.3 \%$ & $\mathbf{7 1 . 7 \%}$ & $\mathbf{8 7 . 3 \%}$ & $68.5 \%$ \\
\hline
\end{tabular}

In the future work, it will be useful to obtain more samples with dirt in order to perform classification of size groups on bigger sets. Besides that, if there is a need, particle texture can be studied to discover new features. Low performance of some classifiers has to be also addressed: either those classifiers should not be used or their parameters must be tuned. Some prior information from the experts might be obtained concerning the frequency of occurrence of the specific dirt types. 


\section{Conclusion}

The present study focuses on the problem of the adaptive classification of dirt particles. An approach for feature selection is described, and relevant features are extracted for classification. The experiments were performed on the whole set and for the size groups of the particles.

It was discovered that for different size groups of dirt different features count. The satisfactory results of classification were obtained for the whole data set. Although the results were slightly worse for size groups, it was shown that the size groups of classes are less overlapping in the feature space. The results show that the critical task is to select the appropriate features. The final tuning for an application can be made by selecting a specific classifier from the best ones and with enough tuning possibilities.

\section{Acknowledgements}

The research was carried out in the "PulpVision" project (TEKES project 70010/10) funded by the European Union and the participating companies. The authors wish to acknowledge the FiberLaboratory in LUT, TEKES, and the companies for their support and collaboration.

\section{References}

1. Duarte, F., Araujo, H., Dourado, A.: An Automatic System for Dirt in Pulp Inspection Using Hierarchical Image Segmentation. Computers \& Industrial Engineering 37, 343-346 (1999)

2. Jones, S., Thomas, R., Awcock, G., Humphrey, K.: Machine vision techniques for ink particle analysis within the paper recycling process. In: Fifth International Conference on Image Processing and its Applications, pp. 682-686 (1995)

3. Parker, S., Chan, J.R.: Dirt Counting in Pulp: An Approach Using Image Analysis Methods. In: Proceedings of the IASTED International Conference on Signal and Image Processing, SIP (2002)

4. Fouladgaran, M., Mankki, A., Lensu, L., Käyhkö, J., Kälviäinen, H.: Automated Counting and Characterization of Dirt Particles in Pulp. In: Bolc, L., Tadeusiewicz, R., Chmielewski, L.J., Wojciechowski, K. (eds.) ICCVG 2010. LNCS, vol. 6375, pp. 166-174. Springer, Heidelberg (2010)

5. Fukunaga, K.: Introduction to Statistical Pattern Recognition. Academic Press, San Diego (1990)

6. The Verity IA Color Image Analysis software. Dirt Counter, http://www.verityia.com/stripscanner.php

7. Kittler, J., Illingworth, J.: On threshold selection using clustering criteria. IEEE Transactions on System, Man, and Cybernetics 12, 652-655 (1985)

8. Figueiredo, M.A.T., Jain, A.K.: Unsupervised learning of finite mixture models. IEEE Transactions on Pattern Analysis and Machine Intelligence 24(3), 381-396 (2002)

9. Theodoridis, S., Koutroumbas, K.: Pattern Recognition. Academic Press, London (1999) 
10. Sezgin, M., Sankur, B.: Survey over image thresholding techniques and quantitative performance evaluation. Journal of Electronic Imaging 13(1), 146-165 (2004)

11. Duda, R.O., Hart, P.E., Stork, D.G.: Pattern Classification. Wiley, Chichester (2001)

12. Freund, Y.: Boosting a weak learning algorithm by majority. Information and Computation 121(2), 256-285 (1995)

13. Pulps - Estimation of dirt and shives - Part 1: Inspection of laboratory sheets by transmitted light. ISO 5350-1:2006 